\title{
Galactose treatment in focal and segmental glomerulosclerosis
}

\author{
Om P. Mishra • Arun K. Singh
}

Received: 20 November 2013 /Revised: 22 November 2013 / Accepted: 12 December 2013 / Published online: 18 January 2014

(C) IPNA 2014

Sirs,

We read with interest the article entitled "Effect of galactose on glomerular permeability and proteinuria in steroid-resistant nephrotic syndrome" by Sgambat et al. recently published in Pediatric Nephrology [1]. In this prospective clinical trial, oral galactose was administered in seven pediatric patients with steroid-resistant nephrotic syndrome for a 16-week period to observe reductions in focal sclerosis permeability factor (FSPF) and proteinuria. Though there was a significant reduction in mean FSPF activity in the post-treatment phase $(0.69 \pm$ 0.11 to $0.35 \pm 0.21, p=0.009$ ), mean urine protein/creatinine (UPC) ratios did not differ significantly between pre- and post-treatment periods $(15.5 \pm 18.9$ vs. $20.8 \pm 25.2 \mathrm{~g} / \mathrm{g}$, respectively). If we look at the data of UPC ratios carefully, it followed non-Gaussian distribution and paired Student's ttest applied for comparison was inappropriate. Therefore, it made the data difficult to interpret. In such data, nonparametric test Wilcoxon signed-rank test should have been applied with calculations of median and interquartile range.

We observed reductions in UPC ratios and an increase in serum albumin levels following oral galactose administration in three patients with focal segmental glomerulosclerosis (FSGS) who were unresponsive to cyclosporine, prednisolone, and ramipril therapy given for 11-14 months. The benefit was demonstrated twice (first course for 90 days and second for 30 days) and deterioration of parameters occurred after discontinuation of galactose. As such, no patients achieved complete remission (UPC $<0.2$ ), but there were definite reductions in UPC ratios in all our three patients [2]. Kopac et al. [3] observed a beneficial effect of galactose in two pediatric patients and the effect persisted for 3 months in one child. However, we observed benefit as long as galactose treatment was continued; implying the effect was durationdependent. In conclusion, oral galactose can be tried as an adjunct therapy to reduce heavy proteinuria in unresponsive FSGS patients.

\section{References}

1. Sgambat K, Banks M, Moudgil A (2013) Effect of galactose on glomerular permeability and proteinuria in steroid-resistant nephrotic syndrome. Pediatr Nephrol 28:2131-2135

2. Mishra OP, Singh AK, Pohl M, Kumar B, Batra VV, Narayan G (2013) Oral galactose in children with focal and segmental glomerulosclerosis: a novel adjunct therapy. Clin Kidney J. doi:10.1093/ckj/sft147

3. Kopac M, Meglic A, Rus RR (2011) Partial remission of resistant nephrotic syndrome after oral galactose therapy. Ther Apher Dial 15: 269-272
O. P. Mishra $(\bowtie) \cdot$ A. K. Singh

Department of Pediatrics, Institute of Medical Sciences, Banaras

Hindu University, Varanasi 221005, India

e-mail: opmpedia@yahoo.co.uk 